\title{
Ultrasmall silicon quantum dots
}

\section{Citation}

Zwanenburg, F. A., A. A. van Loon, G. A. Steele, C. E. W. M. van Rijmenam, T. Balder, Y. Fang, C. M. Lieber, and L. P. Kouwenhoven. 2009. "Ultrasmall Silicon Quantum Dots." Journal of Applied Physics 105 (12) (June 15): 124314. doi:10.1063/1.3155854.

\section{Published Version}

10.1063/1.3155854

\section{Permanent link}

http://nrs.harvard.edu/urn-3:HUL.InstRepos:33464184

\section{Terms of Use}

This article was downloaded from Harvard University's DASH repository, and is made available under the terms and conditions applicable to Other Posted Material, as set forth at http:// nrs.harvard.edu/urn-3:HUL.InstRepos:dash.current.terms-of-use\#LAA

\section{Share Your Story}

The Harvard community has made this article openly available.

Please share how this access benefits you. Submit a story.

Accessibility 


\title{
Ultrasmall silicon quantum dots
}

\author{
F. A. Zwanenburg, ${ }^{1, a)}$ A. A. van Loon, ${ }^{1}$ G. A. Steele, ${ }^{1}$ C. E. W. M. van Rijmenam, ${ }^{1}$ \\ T. Balder, ${ }^{1}$ Y. Fang, ${ }^{2}$ C. M. Lieber, ${ }^{2}$ and L. P. Kouwenhoven ${ }^{1, b)}$ \\ ${ }^{1}$ Kavli Institute of Nanoscience, Delft University of Technology, P.O. Box 5046, 2600 GA Delft, \\ The Netherlands \\ ${ }^{2}$ Department of Chemistry and Chemical Biology, Harvard University, Cambridge, Massachusetts 02138, \\ USA
}

(Received 6 April 2009; accepted 19 May 2009; published online 29 June 2009)

\begin{abstract}
We report the realization of extremely small single quantum dots in $p$-type silicon nanowires, defined by Schottky tunnel barriers with $\mathrm{Ni}$ and NiSi contacts. Despite their ultrasmall size the $\mathrm{NiSi}-\mathrm{Si}-\mathrm{NiSi}$ nanowire quantum dots readily allow spectroscopy of at least ten consecutive holes, and additionally they display a pronounced excited-state spectrum. The Si channel lengths are visible in scanning electron microscopy images and match the dimensions predicted by a model based on the Poisson equation. The smallest dots $(<12 \mathrm{~nm})$ allow identification of the last charge and thus the creation of a single-charge quantum dot. (C) 2009 American Institute of Physics. [DOI: $10.1063 / 1.3155854]$
\end{abstract}

\section{INTRODUCTION}

The observation of quantum states in silicon devices requires small structures because of the relatively high electron and hole effective masses compared to, e.g., InAs or GaAs. Top-down planar silicon devices, ${ }^{1-3}$ produced by etching bulk materials down to nanometer dimensions, often show structural roughness or dopant fluctuations. The corresponding potential fluctuations can result in localization on short length scales, and thus multiple dots connected in series. Nearly all reports so far have been on $\mathrm{Si}$ quantum dots greater than $50 \mathrm{~nm}$, e.g., in $\mathrm{Si}$ metal oxide semiconductor field effect transistors, ${ }^{4-6}$ silicon-on-insulator structures, ${ }^{1-3,7}$ and $\mathrm{Si} / \mathrm{SiGe}$ heterostructures. ${ }^{8,9}$ In these three systems excited states have been observed only recently. ${ }^{10-12}$ Silicon nanowires, on the other hand, naturally provide nanometer scale confinement without etching and have demonstrated clean quantum dot behavior early as $2005 .^{13}$ The latter include excited-state spectroscopy, spin spectroscopy, and a strong indication of the realization of a single-hole dot. We have continued this work and gained a better understanding of the specific system. This has allowed us to realize the first experimental identification of the first four spin states in a silicon quantum dot. ${ }^{14}$ Here we present a thorough investigation of the Si nanowire quantum dot system and the techniques for achieving a single-hole Si nanowire quantum dot, crucial for those results.

We have measured many single quantum dots in $p$-type silicon nanowires, defined by Schottky tunnel barriers with $\mathrm{Ni}$ and NiSi leads. Tunnel barriers defined by Schottky contacts remain fairly constant over a large range of dot occupation numbers. Gate-defined tunnel barriers, however, are much more sensitive to small shifts in the electrostatic field. Hence the quantum dot regime rapidly changes from weakly coupled to strongly coupled, making it nearly impossible to

\footnotetext{
${ }^{a)}$ Electronic mail: floriszwanenburg @ gmail.com.

b) Author to whom correspondence should be addressed. Electronic mail: 1.p.kouwenhoven@tudelft.nl.
}

observe quantum states. We have realized quantum dots shorter than $30 \mathrm{~nm}$, on which we are able to perform spectroscopy of at least ten consecutive holes with pronounced excited states. We can make a reliable estimate of the dot length by comparing the measured backgate capacitance to the capacitance computed with a model based on the Poisson equation. The Si channel lengths visible in scanning electron microscopy (SEM) images match the dimensions predicted by the model. Finally, we emphasize the difficulty of creating a single-hole silicon quantum dot, since many single dots split up in two dots upon reaching the few-hole regime. In our experience only very short dots $(<12 \mathrm{~nm})$ have allowed identification of the last charge in transport measurements.

\section{TWO TYPES OF SI NANOWIRE QUANTUM DOTS}

In this section two approaches for fixing the $\mathrm{Si}$ channel length are compared: (i) $\mathrm{Si}$ nanowires with evaporated $\mathrm{Ni}$ contacts which are defined by electron-beam lithography and (ii) NiSi-Si-NiSi nanowires, fabricated by thermally induced diffusion of $\mathrm{Ni}$ into $\mathrm{Si}$ nanowires. Single-crystal $p$-type $\mathrm{Si}$ nanowires are prepared by a gold nanocluster mediated vapor-liquid-solid process ${ }^{15}$ using silane and diborane as precursor gases with an atomic feed-in ratio of $\mathrm{Si}: \mathrm{B}=4000: 1$. The typical diameter is $7-12 \mathrm{~nm}$, comprising a Si core of 3-8 $\mathrm{nm}$ and a native oxide of $\sim 2 \mathrm{~nm}$. After growth we deposit the nanowires on a highly doped silicon substrate capped with a dry thermal oxide. Predeposited markers allow locating individual nanowires with a SEM and defining contacts by means of electron-beam lithography. The samples are treated with buffered hydrofluoric acid for $5 \mathrm{~s}$ prior to metal deposition to etch off the native $\mathrm{SiO}_{2}$. We deposit $\mathrm{Ni}$ contacts, leaving a Si channel of 50-300 nm uncovered, see Fig. 1(a). In order to make NiSi-Si-NiSi nanowires the samples are annealed in sequential steps of 20-30 s at 380 and $410{ }^{\circ} \mathrm{C}$ to induce radial and longitudinal diffusion of $\mathrm{Ni}$ into the $\mathrm{Si}$ nanowire. From both $\mathrm{Ni}$ contacts a NiSi segment is formed in the nanowires with lengths of 100-150 nm de- 


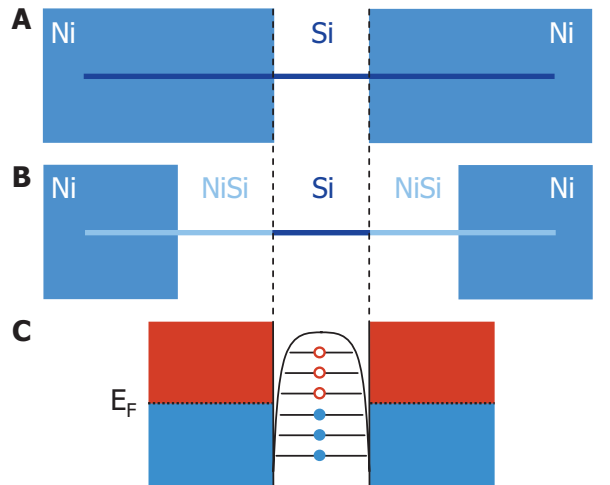

FIG. 1. (Color) Two types of Si nanowire quantum dots. Schematic top view of a Si nanowire quantum dot (a) with $\mathrm{Ni}$ leads and with (b) NiSi leads. Here the Ni has diffused into the Si nanowire during a two-step annealing process. (c) Schematic of the corresponding Schottky tunnel barriers that define the quantum dot. Occupied (empty) hole states are indicated in red (blue).

pending on diameter, temperature, and time. The remaining $\mathrm{Si}$ section is connected to the lithographically defined $\mathrm{Ni}$ contacts by two NiSi leads, see Fig. 1(b). These devices have room-temperature resistances varying from $100 \mathrm{k} \Omega$ to $5 \mathrm{M} \Omega$. Some nanowires are fully transformed into the metal $\mathrm{NiSi}$ having room-temperature resistances of $1-5 \mathrm{k} \Omega$, corresponding to $\rho \sim 10 \mu \Omega \mathrm{cm}$, consistent with values found in NiSi nanowires ${ }^{16}$ and large single crystals. ${ }^{17}$

\section{SINGLE QUANTUM DOTS OF VARYING LENGTHS}

The Ni-Si or NiSi-Si interfaces in each device form the Schottky tunnel contacts between the metallic leads and the $p$-Si quantum dot, see Fig. 1(c). We have measured $6 \mathrm{Ni}-$ $\mathrm{Si}-\mathrm{Ni}$ and $30 \mathrm{NiSi}-\mathrm{Si}-\mathrm{NiSi}$ single quantum dots on substrates with $\mathrm{SiO}_{2}$ thicknesses of 50 and $285 \mathrm{~nm}$. They are cooled down to cryogenic temperatures with a pumped ${ }^{4} \mathrm{He}$ cryostat or a dilution refrigerator. Electrical characterization is carried out by measuring the current from drain to ground while sweeping the bias voltage at the source, $V_{S D}$, and stepping the backgate voltage, $V_{B G}$. In Fig. 2 the differential conductance, $d I / d V_{S D}$, of four devices of varying sizes is plotted versus $V_{S D}$ and $V_{B G}$. We will refer to devices $A$-Ni, $B$-NiSi, $C$-Ni, and $D$-NiSi for the respective figures Figs. 2(a)-2(d).

All four subfigures display diamond-shaped regions in which the current is zero due to Coulomb blockade and the number of holes on the quantum dot, $N$, is fixed. From these Coulomb diamonds we have derived the most important parameters in Table I. In the smallest diamonds the charging energy $E_{C}$ is equal to the addition energy $E_{\text {add }}$. The latter is taken from the height of a Coulomb diamond, indicated as $\Delta V_{S D}$ in Fig. 2(b). The backgate-to-dot capacitance $C_{B G}$ is calculated as $C_{B G}=e / \Delta V_{B G}$, where $\Delta V_{B G}$ is the voltage needed to add a single charge, see Fig. 2(b). The diamond edges have slopes of $-C_{B G} / C_{S}$ and $C_{B G} /\left(C-C_{S}\right)$, where $C_{S}$ $\left(C_{D}\right)$ is the capacitance between the dot and source (drain). ${ }^{18}$ $C$ is the sum of all capacitances to the dot and follows from the charging energy as $e^{2} / C$. If we assume that no other gates have a significant capacitive coupling to the dot, then $C=C_{S}+C_{D}+C_{B G}$. We also compute the gate coupling factor
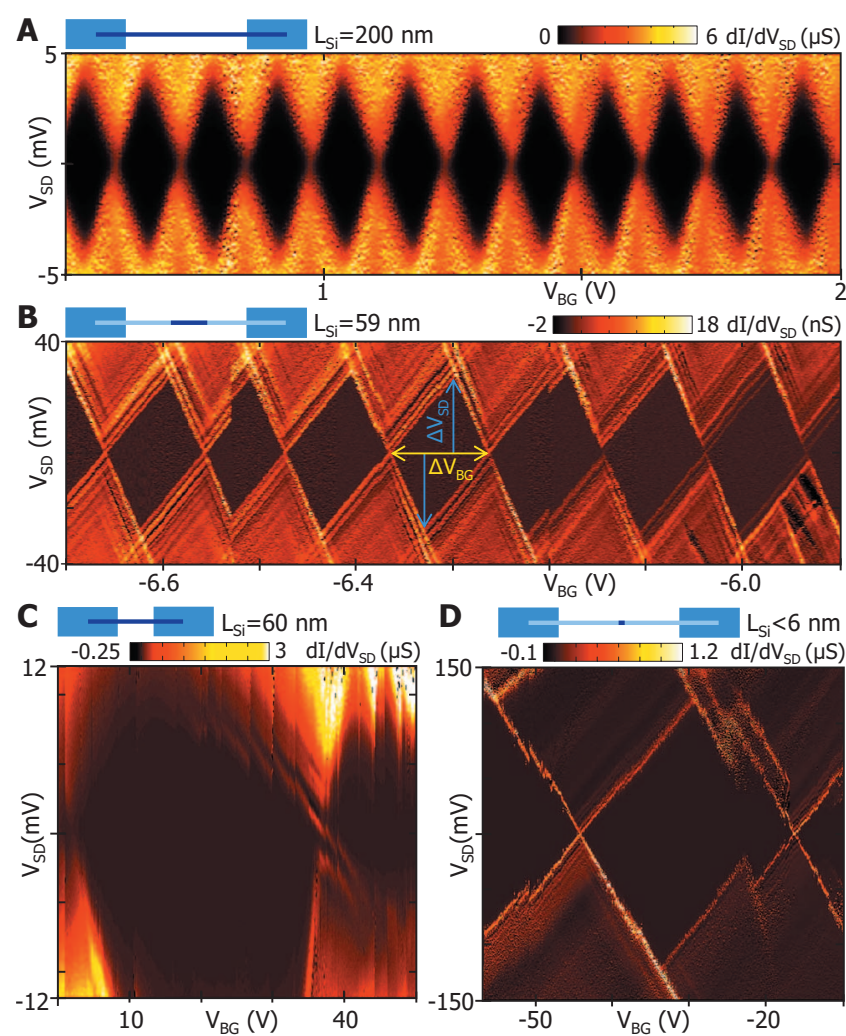

FIG. 2. (Color) Single quantum dots of varying lengths. Stability diagrams of four Si nanowire quantum dots, measured at [(a)-(c)] $2 \mathrm{~K}$ and (d) $20 \mathrm{mK}$. [(a) and (c)] Ni-Si-Ni quantum dots of 200 and $60 \mathrm{~nm}$, as defined by electron-beam lithography. [(b) and (d)] $\mathrm{NiSi}-\mathrm{Si}-\mathrm{NiSi}$ nanowire quantum dots with estimated lengths of 59 and $6 \mathrm{~nm}$, see next figure. The devices $A$-Ni, $C$-Ni, and $D$-NiSi were fabricated on substrates with $\mathrm{SiO}_{2}$ thicknesses of $285 \mathrm{~nm}$ and device $B$-NiSi on $50 \mathrm{~nm}$ thick $\mathrm{SiO}_{2}$.

$\alpha$ as $E_{\text {add }} / \Delta V_{B G}$. The length $L$ of the NiSi-Si-NiSi quantum dots is estimated by means of a capacitance model, see Sec. IV. We approximate the number of charges in these Coulomb diamonds by counting up holes from zero, starting at the backgate voltage at which the dot is emptied at a high voltage bias.

We start by comparing the low temperature measurements on the $\mathrm{Ni}-\mathrm{Si}-\mathrm{Ni}$ quantum $\operatorname{dot} A-\mathrm{Ni}$ and the $\mathrm{NiSi}-\mathrm{Si}-$ $\mathrm{NiSi}$ nanowire quantum dot $B$-NiSi. Both stability diagrams in Figs. 2(a) and 2(b) display a set of uniform Coulomb diamonds. Besides a longer $\mathrm{Si}$ channel, device $A-\mathrm{Ni}$ also has a diameter of $\sim 20 \mathrm{~nm}$, versus $\sim 6 \mathrm{~nm}$ for device $B$-NiSi. The source and drain capacitances of device $A-\mathrm{Ni}$ are about ten times bigger than those of device $B-\mathrm{NiSi}$, because of the order of magnitude difference in volume. Naively, one would expect the same ratio for the backgate capacitances, but they have comparable values. The difference in volume is compensated by the fact that in a NiSi-Si-NiSi nanowire the leads and quantum dot have comparable diameters, and by the difference in $\mathrm{SiO}_{2}$ thickness. The first strongly reduces screening of the backgate compared to lithographically defined leads of device $A$-Ni.

Outside the diamonds of device $B$-NiSi many lines of increased conductance run parallel to the edges, whereas the conductance of device $A-\mathrm{Ni}$ shows no such structure. The origin can be found in the availability of extra channels for transport. Lines ending on the $N$ th diamond are attributed to 
TABLE I. Parameters of the quantum dots of Fig. 2. We extract the capacitances, energies, and $\alpha$-factors from the bias spectroscopies in Fig. 2. For Figs. 2(c) and 2(d) we have used the only full Coulomb diamond to determine the parameters. $N$ is approximated by counting up holes from zero, starting at the backgate voltage at which the dot is emptied at a high source drain bias voltage.

\begin{tabular}{lclcccccc}
\hline \hline Device & $\begin{array}{c}L \\
(\mathrm{~nm})\end{array}$ & $\begin{array}{c}C_{B G} \\
(\mathrm{aF})\end{array}$ & $\begin{array}{c}C_{S} \\
(\mathrm{aF})\end{array}$ & $\begin{array}{c}C_{D} \\
(\mathrm{aF})\end{array}$ & $\begin{array}{c}\alpha \\
(\mathrm{eV} / \mathrm{V})\end{array}$ & $\begin{array}{c}E_{C} \\
(\mathrm{meV})\end{array}$ & $\begin{array}{c}\Delta E \\
(\mathrm{meV})\end{array}$ & $N$ \\
\hline$A-\mathrm{Ni}$ & 200 & 1.3 & 21.2 & 16.9 & 0.033 & 4.1 & $<k_{B} T$ & 800 \\
$B-\mathrm{NiSi}$ & 59 & 1.6 & 2.0 & 2.2 & 0.28 & 28.4 & 1.1 & 40 \\
$C-\mathrm{Ni}$ & 60 & 0.006 & 6.9 & 1.0 & 0.001 & 20.0 & 2 & 30 \\
$D-\mathrm{NiSi}$ & $<6$ & 0.007 & 0.5 & 0.8 & 0.005 & 120 & 120 & 1 \\
\hline \hline
\end{tabular}

the excited states of the $N$ th hole. ${ }^{18}$ The fact that excited states are visible is a direct consequence of the smaller size of quantum dot $B$-NiSi because the magnitude of excited states generally increases with decreasing dot size. The small energy level spacing of the $200 \mathrm{~nm}$ quantum dot (device $A-\mathrm{Ni}$ ) is washed out by the thermal energy, $k_{B} T \sim 0.2 \mathrm{meV}$. However, in the case of the $59 \mathrm{~nm}$ dot (device $B$-NiSi) the observed excited states are about $1 \mathrm{meV}$, bigger than $k_{B} T$. We have also fabricated a $\mathrm{Ni}-\mathrm{Si}-\mathrm{Ni}$ quantum dot of the same size as device $B$-NiSi, which displays excited states of roughly $2 \mathrm{meV}$, see Fig. 2(c). Figure 2(d) shows a stability diagram of the shortest $\mathrm{NiSi}-\mathrm{Si}-\mathrm{NiSi}$ dot we measured, with a length below $6 \mathrm{~nm}$. The excited state ending on the leftmost diamond is about $120 \mathrm{meV}$, and thus confirms the extremely small size of the dot.

Often hard-wall and harmonic potential wells are used to match measured orbital states to a theoretical prediction. In case of a hard-wall potential in a one-dimensional system the energy difference between two levels is $E_{n+1}-E_{n}=(2 n$ $+1) \hbar^{2} \pi^{2} / 2 m_{\mathrm{eff}} L^{2},{ }^{19}$ where $m_{\mathrm{eff}}$ is the effective mass and $L$ the size of the quantum well. The predicted increase in level spacing with the number of charges has in practice seldom been observed and is clearly not present in our devices. On the other hand, for a Schottky barrier harmonic oscillator potential the level spacing, $\hbar \omega_{0}$, is constant and can be estimated from $V=1 / 2 m_{\mathrm{eff}} \omega_{0}^{2}(L / 2)^{2}$, where $V$ is the Schottky barrier height. ${ }^{20}$ However, for a $200 \mathrm{~nm}$ quantum dot (device $A$-Ni) the level spacing would be $3.6 \mathrm{meV}$, much greater than $k_{B} T$. Here we use an effective hole mass of $0.59 m_{0}$ (Ref. 21) and a Ni-Si Schottky barrier height of $0.51 \mathrm{eV}$. Of all our devices, only dots shorter than $70 \mathrm{~nm}$ show excited states, whereas according to the Schottky barrier harmonic model the level spacing of an $80 \mathrm{~nm}$ dot should be about $9 \mathrm{meV}$, and thus easily observable.

Since neither model gives a satisfactory description we use a more general expression for the quantum confinement energy as $\Delta E_{\text {conf }} \sim h^{2} / m_{\text {eff }} L^{2}$. For a $200 \mathrm{~nm}$ quantum dot $\Delta E_{\text {conf }}=0.13 \mathrm{meV}$, smaller than $k_{B} T$ as observed. The confinement energy of a $59 \mathrm{~nm}$ dot is $1.5 \mathrm{meV}$, bigger than $k_{B} T$, and it corresponds well to the observed excited-state lines of $1.1 \mathrm{meV}$. In case of a dot of 4-6 nm, $\Delta E_{\text {conf }}$ $=140-320 \mathrm{meV}$, fairly close to the measured excited state of $120 \mathrm{meV}$. We find that the above expression for the confinement energy gives a reliable estimate for the level spacing in our system.

The comparable source and drain capacitances of devices $B$-NiSi and $C$-Ni confirm the similarity of the dot sizes.
However, the backgate voltage needed to add one hole to dot $C$-Ni is about $30 \mathrm{~V}$, a factor of 300 more than for device $B$-NiSi. Performing spectroscopy of four consecutive holes thus requires a change in backgate voltage of more than 100 $\mathrm{V}$. This is problematic since sweeping $V_{B G}$ over a large range increases the chance of gate leakage and charge rearrangements on the $\mathrm{SiO}_{2}$. Both can lead to potential fluctuations and thus unstable devices, see Fig. 2(c). On the other hand, we have measured 22 single $\mathrm{NiSi}-\mathrm{Si}-\mathrm{NiSi}$ nanowires shorter than $50 \mathrm{~nm}$. Even NiSi-Si-NiSi quantum dots with channel lengths between 10 and $20 \mathrm{~nm}$ need less than $2 \mathrm{~V}$ to add a single charge and thus readily allow spectroscopy of at least ten holes. Evidently, NiSi-Si-NiSi nanowires are more suitable to measure short quantum dots than $\mathrm{Ni}-\mathrm{Si}-\mathrm{Ni}$ nanowires.

This is underlined by the results on device $D$-NiSi: just like in device $C-\mathrm{Ni}$, the peak-to-peak distance is $30 \mathrm{~V}$ on the
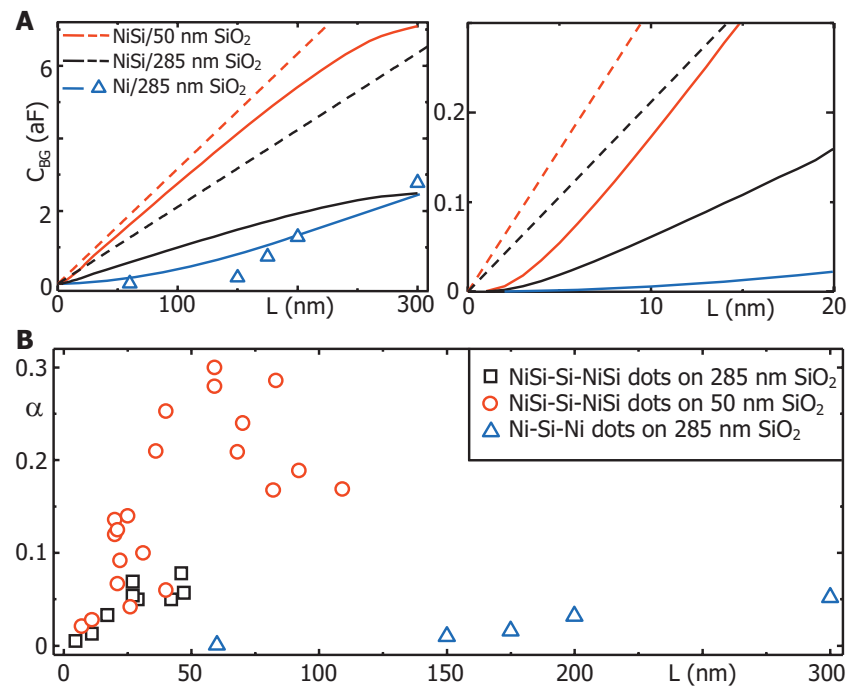

FIG. 3. (Color) Capacitances and dot lengths. (a) Computed values of the backgate capacitance for $\mathrm{NiSi}-\mathrm{Si}-\mathrm{NiSi}$ dots on substrates with $50 \mathrm{~nm}$ (red) and $285 \mathrm{~nm}$ (black) thick $\mathrm{SiO}_{2}$ and $\mathrm{Ni}-\mathrm{Si}-\mathrm{Ni}$ dots on substrates with $285 \mathrm{~nm}$ thick $\mathrm{SiO}_{2}$ (blue). Dashed lines are calculated with a cylinder-on-plate model and straight lines with a model based on the Poisson equation, which includes screening. Blue triangles are measured backgate capacitances of $\mathrm{Ni}-\mathrm{Si}-\mathrm{Ni}$ quantum dots. Right panel shows a zoom at dot lengths below 20 $\mathrm{nm}$. The cylinder-on-plate model does not discriminate between $\mathrm{Ni}-\mathrm{Si}-\mathrm{Ni}$ and $\mathrm{NiSi}-\mathrm{Si}-\mathrm{NiSi}$ quantum dots. (b) Experimentally found gate coupling factors vs dot length for all types of quantum dots. The Ni-Si-Ni dots have much smaller $\alpha$-values than $\mathrm{NiSi}-\mathrm{Si}-\mathrm{NiSi}$ dots, as a result of stronger screening of the electric field induced by the backgate. We have measured one Ni-Si-Ni dot of $840 \mathrm{~nm}$ length with $C_{B G}=14.6 \mathrm{aF}$ and $\alpha=0.27$ [off scale in (a) and (b)]. 


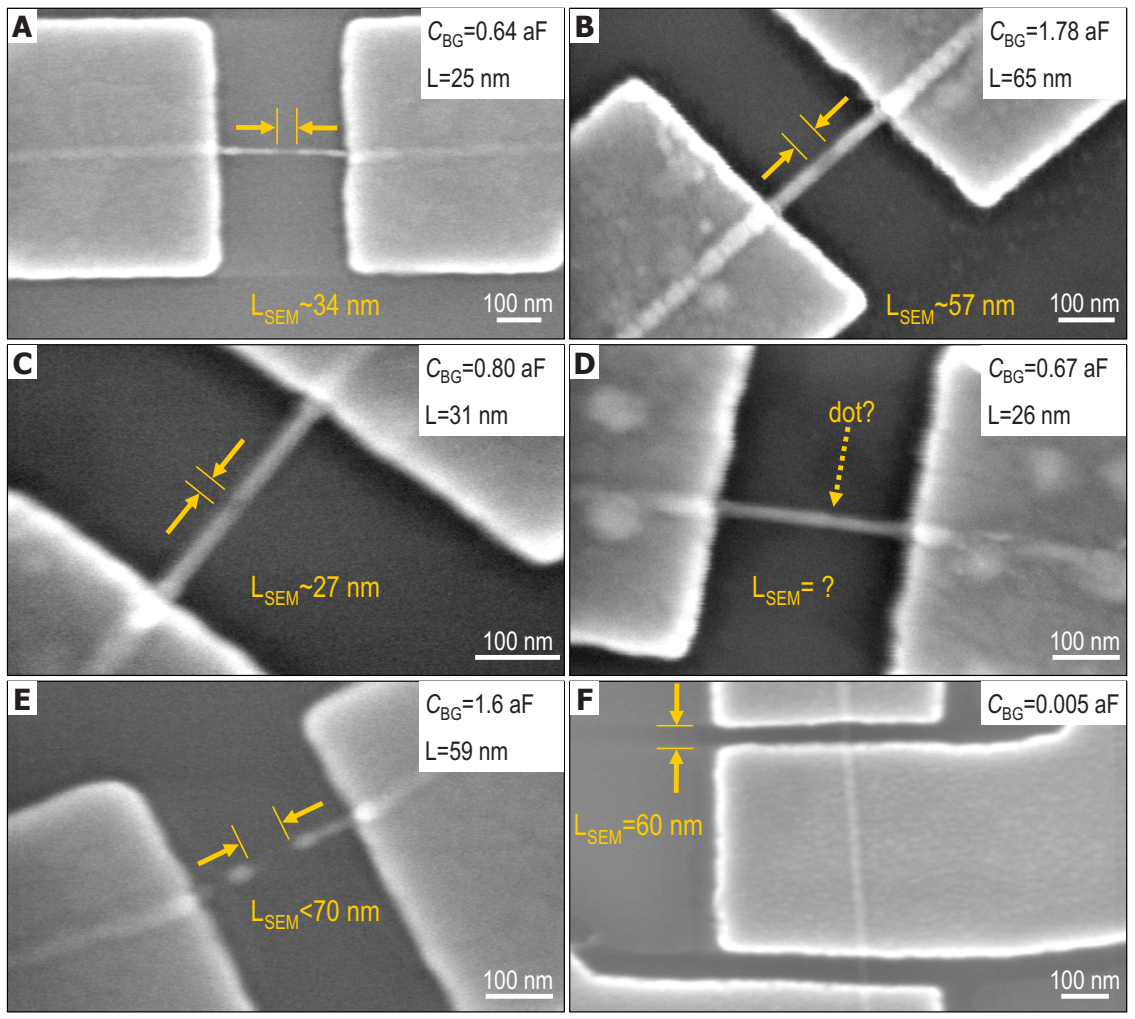

FIG. 4. (Color online) Dot lengths in SEM. SEM images of five different $\mathrm{NiSi}-\mathrm{Si}-\mathrm{NiSi}$ nanowire quantum dots [(a)-(e)] measured on substrates with $50 \mathrm{~nm} \mathrm{SiO}$ and one Ni-Si-Ni quantum dot (f) measured on a substrate with $285 \mathrm{~nm} \mathrm{SiO}$. The image in (d) does not reveal a distinct Si segment. The device in (e) blew up after the measurements, but the remaining $\mathrm{NiSi}$ gives an upper bound for $L_{\mathrm{SEM}}$ of 70 $\mathrm{nm}$. Measurements of the devices in (c), (e), and (f) are shown in, respectively, Ref. 14 and Figs. 2(b) and 2(c). Insets: the backgate capacitance deduced from the measurements and the corresponding model length, see Fig. 3.

backgate, despite the fact that the dot in device $D$-NiSi is more than an order of magnitude shorter. Also, the stability of device $D$-NiSi compared to device $C$-Ni over this gate voltage range is striking and has allowed us to resolve the Zeeman energy of the first hole, see supporting information in Ref. 14.

The results in Fig. 2 show the realization of $\mathrm{Ni}-\mathrm{Si}-\mathrm{Ni}$ and $\mathrm{NiSi}-\mathrm{Si}-\mathrm{NiSi}$ nanowire quantum dots of lengths varying from 4 to $200 \mathrm{~nm}$. The first type provides strong control over the size of the quantum dots down to $50 \mathrm{~nm}$ channel lengths, whereas the latter suffer only lightly from screening at the $\mathrm{NiSi}-\mathrm{Si}$ junctions. A drawback of $\mathrm{NiSi}-\mathrm{Si}-\mathrm{NiSi}$ quantum dots is the variation in $\mathrm{Ni}$ diffusion per device, and therefore the less precise control of the dot length.

\section{CAPACITANCES AND DOT LENGTHS}

A cylinder-on-plate model can be used to connect the measured backgate-to-dot capacitance to the Si dot length as $C_{B G}=2 \pi \epsilon_{0} \epsilon_{r} L / \ln (2 t / r)$, where $t$ is the backgate distance and $r$ is the nanowire radius, see, e.g., Ref. 22. In this model $C_{B G}$ increases linearly with the nanowire length, see Fig. 3(a). However, the capacitance is smaller in real devices because the metallic contacts screen the electric field induced by the backgate. To include these geometric screening effects, we calculate the capacitance of the dot to the backgate using a numerical finite difference solution of the Poisson equation that includes the full three-dimensional geometry of the device. $^{23}$

With this Poisson solver we have calculated the backgate-to-dot capacitance of the Si nanowire devices used in this research, as we have sketched in Figs. 1(a) and 1(b). In case of the $\mathrm{Ni}-\mathrm{Si}-\mathrm{Ni}$ devices we varied the $\mathrm{Ni}$ contact separation from 0 to $300 \mathrm{~nm}$, connected to each other by a 6 $\mathrm{nm}$ diameter $\mathrm{Si}$ nanowire. For the $\mathrm{NiSi}-\mathrm{Si}-\mathrm{NiSi}$ nanowire devices we have used a constant distance of $300 \mathrm{~nm}$ between the $\mathrm{Ni}$ contacts which sandwich the nanowire with a $\mathrm{Si}$ (NiSi) diameter of $6 \mathrm{~nm}(8 \mathrm{~nm})$. We have varied the length of the $\mathrm{Si}$ dot from 0 to $300 \mathrm{~nm}$, resulting in two NiSi leads varying from 150 to $0 \mathrm{~nm}$. The computed $C_{B G}$ versus the $\mathrm{Si}$ dot length has three regimes for $\mathrm{NiSi}-\mathrm{Si}-\mathrm{NiSi}$ nanowires, see Fig. 3(a): (i) For dot sizes between 6 and $250 \mathrm{~nm} C_{B G}$ increases proportionally with the length by roughly $27 \mathrm{aF} / \mu \mathrm{m}$ (9 $\mathrm{aF} / \mu \mathrm{m})$ on substrates with $50 \mathrm{~nm}(285 \mathrm{~nm}) \mathrm{SiO}_{2}$. (ii) In case of $\mathrm{Si}$ dot lengths above $250 \mathrm{~nm}$ the slope of $C_{B G}(L)$ becomes less steep due to screening by the wide Ni contacts. (iii) Below $6 \mathrm{~nm}$ the dot length becomes comparable to the diameter and the electric field lines from the backgate are screened by the NiSi leads, see the zoom in the right panel of Fig. 3(a).

In case of $\mathrm{Ni}-\mathrm{Si}-\mathrm{Ni}$ dots we have performed calculations for substrates with $285 \mathrm{~nm} \mathrm{SiO}_{2}$, see Fig. 3(a). At a channel length of $300 \mathrm{~nm}$ the calculated capacitance is the same as for NiSi-Si-NiSi dots because the NiSi leads in the latter have been reduced to $0 \mathrm{~nm}$ and the geometries are thus equal. Below $300 \mathrm{~nm}$ the capacitance of Ni-Si-Ni dots drops rapidly, whereas the values of dots with NiSi leads decrease slowly. At dot lengths below $40 \mathrm{~nm}$ there is more than a factor of 5 difference between the capacitances of $\mathrm{NiSi}-\mathrm{Si}$ $\mathrm{NiSi}$ and $\mathrm{Ni}-\mathrm{Si}-\mathrm{Ni}$ dots. The measured capacitance values of the latter correspond well to the computed values, and the model thus gives a reliable estimate of the dot length. For these small dots the capacitances computed by the cylinderon-plate model are far off realistic values.

Along these lines we can connect the measured backgate capacitance of a NiSi-Si-NiSi device to a dot length. We calculate the capacitance from Coulomb diamonds at high 
hole numbers, where the tunnel barriers are thinnest and accordingly the size of the orbital wave functions corresponds best to the Si channel length. Scanning electron micrographs reveal silicide segments as bright regions which sandwich a darker section of Si, see Fig. 4. When the channel length measured in SEM images is compared to the length predicted by the model we obtain an accuracy within $30 \%$ in different devices. In some cases we cannot exactly determine the dot in the SEM images, e.g., Figs. 4(d) and 4(e). Additionally we have derived the gate coupling factors from the measurements and plotted them versus dot length in Fig. 3(c). In this geometry the gate coupling factor is a good measure for the strength of the screening. This explains the distinct difference in $\alpha$-values between $\mathrm{NiSi}-\mathrm{Si}-\mathrm{NiSi}$ dots and $\mathrm{Ni}-\mathrm{Si}-\mathrm{Ni}$ dots. In case of the latter enormous screening due to the wide $\mathrm{Ni}$ contacts strongly complicates measuring $\mathrm{Si}$ quantum dots smaller than $50 \mathrm{~nm}$. Clearly we have been able to fabricate many $\mathrm{NiSi}-\mathrm{Si}-\mathrm{NiSi}$ dots with lengths below $50 \mathrm{~nm}$, which readily allow spectroscopy of at least ten holes.

\section{THE FEW-HOLE REGIME}

Finally, we have succeeded at isolating a single hole on a Si quantum dot by driving the gate voltage to more positive values. In practice it turns out that most devices split up into two dots before we reach the last charge. This can be understood by small potential fluctuations in the bottom of the $\mathrm{Si}$ potential well resulting in the formation of an extra tunnel barrier at lower hole numbers. We attribute the local potential perturbations to impurities or defects in the environment of the dot. So far, only three very short dots have allowed positive identification of the last charge, see Refs. 13 and 14. The estimated dot lengths of these devices are 5, 9, and 12 $\mathrm{nm}$. Shorter channels increase the chance to get a single-hole dot. First for statistical reasons: the chance of having, e.g., an impurity or a defect right next to the dot decreases with the channel length. Second, if the confining potential is determined by two Schottky barriers without a flat valence band in between, the quantum well is less sensitive to modulations in the electrostatic potential due to the steepness of the bent valence band.

\section{CONCLUSION}

In conclusion, we have demonstrated the realization of single $\mathrm{Si}$ quantum dots with $\mathrm{Ni}$ and $\mathrm{NiSi}$ contacts. In Ni$\mathrm{Si}-\mathrm{Ni}$ quantum dots we have strong control over the size of the quantum dots down to $50 \mathrm{~nm}$ channel lengths. However, the metallic leads severely screen electric field lines induced by a backgate, whereas the leads and quantum dot have comparable diameters in a $\mathrm{NiSi}-\mathrm{Si}-\mathrm{NiSi}$ nanowire. Screening from contacts at $\mathrm{NiSi}-\mathrm{Si}$ junctions is very small compared to
$\mathrm{Ni}-\mathrm{Si}$ junctions and thus the backgate has a stronger capacitive coupling. An extra advantage is that this technique allows the formation of dots shorter than $30 \mathrm{~nm}$ with pronounced excited states and the observation of single-hole dots.

\section{ACKNOWLEDGMENTS}

This work was supported by the Dutch Organization for Fundamental Research on Matter (FOM), the Netherlands Organization for Scientific Research (NWO), and NanoNed, a national nanotechnology program coordinated by the Dutch Ministry of Economic Affairs. C.M.L. acknowledges Samsung Electronics and a contract from MITRE Corporation for support of this work.

${ }^{1}$ A. Tilke, R. H. Blick, H. Lorenz, J. P. Kotthaus, and D. A. Wharam, Appl. Phys. Lett. 75, 3704 (1999).

${ }^{2}$ L. P. Rokhinson, L. J. Guo, S. Y. Chou, and D. C. Tsui, Phys. Rev. B 63, 035321 (2001).

${ }^{3}$ E. G. Emiroglu, D. G. Hasko, and D. A. Williams, Appl. Phys. Lett. 83, 3942 (2003).

${ }^{4}$ G. M. Jones, B. H. Hu, C. H. Yang, M. J. Yang, R. Hajdaj, and G. Hehein, Appl. Phys. Lett. 89, 073106 (2006).

${ }^{5}$ F. Simmel, D. Abusch-Magder, D. A. Wharam, M. A. Kastner, and J. P. Kotthaus, Phys. Rev. B 59, R10441 (1999).

${ }^{6}$ A. Fujiwara, H. Inokawa, K. Yamazaki, H. Namatsu, Y. Takahashi, N. M. Zimmerman, and S. B. Martin, Appl. Phys. Lett. 88, 053121 (2006).

${ }^{7}$ M. Hofheinz, X. Jehl, M. Sanquer, G. Molas, M. Vinet, and S. Deleonibus, Appl. Phys. Lett. 89, 143504 (2006).

${ }^{8}$ M. R. Sakr, H. W. Jiang, E. Yablonovitch, and E. T. Croke, Appl. Phys. Lett. 87, 223104 (2005).

${ }^{9}$ L. J. Klein, D. E. Savage, and M. A. Eriksson, Appl. Phys. Lett. 90, 033103 (2007).

${ }^{10}$ S. J. Angus, A. J. Ferguson, A. S. Dzurak, and R. G. Clark, Nano Lett. 7, 2051 (2007).

${ }^{11}$ H. Liu, T. Fujisawa, H. Inokawa, Y. Ono, A. Fujiwara, and Y. Hirayama, Appl. Phys. Lett. 92, 222104 (2008).

${ }^{12}$ N. Shaji, C. B. Simmons, M. Thalakulam, L. J. Klein, H. Qin, H. Luo, D. E. Savage, M. G. Lagally, A. J. Rimberg, R. Joynt, M. Friesen, R. H. Blick, S. N. Coppersmith, and M. A. Eriksson, Nat. Phys. 4, 540 (2008).

${ }^{13}$ Z. Zhong, Y. Fang, W. Lu, and C. M. Lieber, Nano Lett. 5, 1143 (2005).

${ }^{14}$ F. A. Zwanenburg, C. E. W. M. van Rijmenam, Y. Fang, C. M. Lieber, and L. P. Kouwenhoven, Nano Lett. 9, 1071 (2009).

${ }^{15}$ R. S. Wagner and W. C. Ellis, Appl. Phys. Lett. 4, 89 (1964).

${ }^{16}$ Y. Wu, J. Xiang, C. Yang, W. Lu, and C. M. Lieber, Nature (London) 430 , 61 (2004).

${ }^{17}$ B. Meyer, U. Gottlieb, O. Laborde, H. Yang, J. C. Lasjaunias, A. Sulpice, and R. Madar, J. Alloys Compd. 262, 235 (1997).

${ }^{18}$ R. Hanson, L. P. Kouwenhoven, J. R. Petta, S. Tarucha, and L. M. K. Vandersypen, Rev. Mod. Phys. 79, 1217 (2007).

${ }^{19}$ L. P. Kouwenhoven, C. M. Marcus, P. L. McEuen, S. Tarucha, R. M. Westervelt, and N. S. Wingreen, NATO ASI Ser., Ser. E 345, 16 (1997).

${ }^{20}$ P. D. Jarillo-Herrero, S. Sapmaz, C. Dekker, L. P. Kouwenhoven, and H. S. J. van der Zant, Nature (London) 429, 389 (2004).

${ }^{21}$ M. A. Green, J. Appl. Phys. 67, 2944 (1990).

${ }^{22}$ R. Martel, T. Schmidt, H. R. Shea, T. Hertel, and P. Avouris, Appl. Phys. Lett. 73, 2447 (1998).

${ }^{23} \mathrm{~W}$. H. Press and W. T. Vetterling, Numerical Recipes in C: The Art of Scientific Computing, 2nd ed. (Cambridge University Press, New York, 1993), p. 866. 